\title{
PSO and Kalman Filter-Based Node Motion Prediction for Data Collection from Ocean Wireless Sensors Network with UAV
}

\author{
Tu Dac Ho ${ }^{1}$, Esten Ingar Grøtli ${ }^{2}$, Tor Arne Johansen ${ }^{3}$
}

\begin{abstract}
In this paper, we consider a wireless sensor network of nodes at the sea surface drifting due to wind and sea currents. In our scenario an Unmanned Aerial Vehicle (UAV) will be used to gather data from the sensor nodes. The goal is to find a flyable path which is optimal in terms of sensor node energy consumption, total channel throughput between the UAV and sensor nodes, flight time for the UAV and frequency of the node visits by the UAV. Finally, the path should also be optimal concerning node position estimation uncertainty. A Kalman Filter (KF) is used to estimate the nodes motions and Particle Swarm Optimization (PSO) is the method used to calculate the UAV path taking all of these objectives into account. The proposed node tracking aware path planning solution is compared to two other scenarios: One where the path planning is based on full knowledge of the node positions at all times, and one where path planning is based on the last known positions of the nodes.
\end{abstract}

\section{INTRODUCTION}

Wireless sensing nodes in this paper are assumed to have limited battery capacity, i.e. they cannot harvest energy from their surroundings. These nodes, however, can sense and determine some environmental parameters such as the spatial and temporal extent of water pollution, fields of toxic algae, chlorophyll concentrations [1], salinity, turbidity, $\mathrm{pH}$ [2], oxygen density or fish and mammal activity. In some applications, it is necessary to establish a connected network of nodes to provide full functionality, for example, in online data collection, real-time data analysis and for triggered event capability. Such networks will have a high energy consumption to maintain a fully connected network, which consequently leads to a shorter lifetime for the nodes and the network.

In other scenarios, distributed wireless sensor networks are deployed to large areas because the measurements (for instance of salinity or chemical components in sea water) vary substantially between different locations. To make such wide area networks with many sensors permanently connected, will be costly in terms of energy consumption. For in-situ sensor networks deployed in extreme or remote areas, both the maintenance and data collection can be very challenging.

Unmanned Aerial Vehicle (UAV) technologies have spread widely and become popular in various fields. The most noticeable benefits compared with conventional manned vehicles are low cost, improved safety for humans, and easy deployment. There are many applications in wireless communications

\footnotetext{
${ }^{1}$ T. D. Ho is with Department of Electrical Engineering, Faculty of Engineering Science and Technology, UiT - The Arctic University of Norway $\{\mathrm{Tu} . \mathrm{D} . \mathrm{Ho}\}$ at uit.no

${ }^{2}$ E. I. Grøtli is with Department of Cybernetics and Mathematics, SINTEF Digital, Trondheim, Norway \{esteningar.grotli\} at sintef. no

${ }^{3}$ T. A. Johansen is with the Center for Autonomous Marine Operations and Systems (AMOS), Department of Engineering Cybernetics, Norwegian University of Science and Technology, O. S. Bragstads plass 2D, 7491 Trondheim, Norway $\{$ tor.arne.johansen\} at itk.ntnu.no
}

networks that employ UAVs to extend the range of communications, facilitate broadband $5 \mathrm{G}$ network and extend cellular mobile network assisted by a swarm of small UAVs [3], or to collect data from a wide area network in remote areas or harsh environment [4].

\section{CONTRIBUTIONS AND CONSIDERED SCENARIOS}

In the literature, see for instance [3], [5], [6] and [4] it has commonly been considered that the wireless sensor nodes are static or have limited mobility. In other words, the assumption is that the node's positions are static or known exactly and can be used for path planning. If the nodes are deployed and free to drift on the ocean surface, this assumption will no longer be valid: It is typically too costly or impractical to have permanent connections with the nodes to receive updates on their positions as they drift with ocean currents. The main objective is to relax the above mentioned assumptions. Our main contribution is incorporating a KF in the path planning, and where the KF is used to estimate and predict the nodes positions. The nodes positions can be measured when the UAV is within communication range. We will also investigate the case where it is possible to receive additional measurements of the ocean currents. To assess the approaches we will compare the use of $\mathrm{KF}$ for estimating the nodes positions (Scenario 3 ) with two other scenarios. All three scenarios are described below.

\section{A. Scenario 1: Full sensor node position information}

In this scenario, it is assumed that any information about the nodes is available to the UAV for path planning at all times. It means that the UAV will always get the most updated information about the new positions of the nodes and it is expected that it would be the ideal case which gives the best result. However, due to the movement of the nodes this would require the nodes to repeatedly communicate their position to the UAV. This would be undesirable for two reasons: The additional communication with the UAV will drain the sensor nodes for energy, and the nodes would possibly need additional equipment (satellite communication equipment) to communicate over long distances. If the sensors and the UAV are fully connected, the sensor nodes positions can be relayed via other nodes to the UAV, but this would still decrease the lifetime of the sensor nodes because of high path loss for communications between the nodes.

\section{B. Scenario 2: Range-limited position measurements}

In this scenario the UAV has only information about the new position of the nodes when it is within communication range, and would therefore use the last known position of the sensor nodes for planning. When the UAV can communicate with the nodes, the flight path of the UAV can be updated. The 
drawback is that the nodes may have moved a long distance from the previously known position, which means that the UAV will have to spend more time gathering data from the sensor network. In the worst case, a sensor node may even be "lost", as its new position brings it outside the communication range of the UAV, given that it flies to the last known position of the sensor node. In this case, the UAV can start to search for the nodes, but this is considered outside the scope of this paper.

\section{Scenario 3: Estimation-based position information}

In this scenario the UAV uses a KF to estimate and predict the position of the sensor nodes. Using a model of the environment based on wind and ocean current predictions, the UAV can estimate the new positions of the sensor nodes, based on the nodes' previous location information and the new measurements every time it can communicate with a sensor node.

\section{Comparison of scenarios}

For a fair comparison regarding node energy consumption in these scenarios, we consider the same radio communication link for all of the cases. Only in the first scenario, an adjustable transmitting power for the radio signal is applied to ensure that the UAV can always communicate with sensor nodes when needed. When the distance between the UAV and a sensor node is larger than the maximal communication range (i.e. at the initial transmission power), the transmission power is adjusted to ensure the communications in this case. In the two other cases, a communication link between the UAV and a sensor node is established only when they are in range. This paper will implement these scenarios and compare their performances.

\section{MODELING}

\section{A. Motion model of sensor nodes}

Consider $n$ wireless sensor nodes. Out of simplicity, we will assume that the position vector $x_{i} \in \mathbb{R}^{2}$ of node $i \in$ $\{1,2, \ldots, n\}$ is given by the differential equation $\dot{x}_{i}=\theta+w_{i}$, with initial conditions $x_{i}(0) \in \mathbb{R}^{2}$, and where $\theta \in \mathbb{R}^{2}$ is the velocity vector of the environmental influences (sea-currents and wind) in the East and North directions, and where $w_{i} \in \mathbb{R}^{2}$ is a vector of random Gaussian white noise. We assume that the environmental influences are described by $\dot{\theta}=w_{\theta}$ where $w_{\theta} \sim$ $\mathcal{N}\left(0, Q_{\theta}\right)$ with $Q_{\theta}$ being the covariance. We also assume that the nodes have the same velocity as they are deployed in the same region with small variations in weather and currents. The system of differential equations describing the motion of all the sensor nodes and the constant velocity vector can therefore be given by

$$
\dot{x}=A x+w:=\left[\begin{array}{cc}
0_{2 \times 2 n} & I_{2 \times 2} \\
\cdots & \cdots \\
0_{2 \times 2 n} & I_{2 \times 2} \\
0_{2 \times 2 n} & 0_{2 \times 2}
\end{array}\right] x+w
$$

where $x=\left(x_{1}^{\top}, x_{2}^{\top}, \ldots, x_{n}^{\top}, \theta\right)^{\top} \quad$ and $w=$ $\left(w_{1}^{\top}, w_{2}^{\top}, \ldots, w_{n}^{\top}, w_{\theta}\right)^{\top}$

\section{B. Measurement models}

We consider two different sensor models. In one case, the sensing system gets some information about the speeds of the wind and sea-currents affecting the sensor network, for instance through weather forecasts or other sensing devices. In the other case, we will assume that no such measurements are available, but that the information to some extent can be estimated based on the measured position of the sensors under the assumption that environmental effects are slowly varying or constant.

1) Measurement model without velocity vector measurements: We will assume that the position of the nodes can only be measured when the UAV are within communication distance of the nodes. This is modelled as

$$
y_{i}\left(t_{k}\right)=x_{i}\left(t_{k}\right)+v_{i}\left(t_{k}\right) \quad \text { if } \quad\left\|p\left(t_{k}\right)-x_{i}\left(t_{k}\right)\right\| \leq L_{\max }
$$

where $t_{k}$ is a specific time instance, $y_{i} \in \mathbb{R}^{2}$ is a measurement of the position of node $i \in\{1, \ldots, n\}$ with some additive multivariate zero-mean normal distributed noise with covariance $R_{i}$, that is $v_{i} \sim \mathcal{N}\left(0, R_{i}\right)$. Furthermore, $p$ is the position of the UAV and $L_{\max }$ (which will be explained in details in Section III-C) is the maximal communication distance for the system. This $L_{\max }$ is introduced purely for simplicity in simulating our scenario. The sensors normally listen for a broadcast signal from the UAV and periodically transmit its position along with other sensing data when their actual distance at the transmitting time is less than $L_{\max }$. Also out of simplicity, we will assume that the sensors are equipped with a GPS, the same type, and use the same data length each time they transmit. We emphasize that it is possible to develop a more complex network with different kinds of sensors using different data lengths. Notice also that these are measurements that depend on the relative distance between the UAV and the sensor nodes, and are therefore asynchronous. The UAV will estimate the nodes position which is implemented in a KF.

2) Measurement model with velocity vector measurements: In this case, we will assume that we have the measurement model (2), and in addition

$$
y_{\theta}=\theta+v_{\theta}
$$

so that $y_{\theta} \in \mathbb{R}^{2}$ is a measurement of the environmental effects with some additive multivariate zero-mean normal distributed noise with covariance $R_{\theta}$, that is $v_{\theta} \sim \mathcal{N}\left(0, R_{\theta}\right)$. We can assume that these measurements are only available when the UAV is within communication distance of some weather station or similar, just as in (2), or they are available at a certain refresh rate.

\section{Radio range and transmitting power}

This section focuses on the maximal radio propagation distance and an adjustable transmitting power in communications between the sensor nodes and the UAV. To implement communications between sensor nodes and the UAV in the three cases of Section II, there are only two different situations regarding communication coverages. They are a) The nodes always transmit at a fixed power level and communications occurs within the maximal radio range (i.e. Scenarios 2 \& 3) and $b$ ) the nodes can adjust the transmission power to maintain a continuous connection with the UAV (i.e. Scenario 1). The 
former might not ensure that the UAV always gets the node's location information and sensing data but it will use less of the nodes' energy. The latter secures communication links with the UAV but it causes more energy consumption at the nodes.

1) Maximal radio coverage: First, the receiving radio signal strength $P r_{\text {us }}$ between the UAV and a sensor $s$ can be found from:

$$
P r_{\mathrm{us}}=P t_{0}+G_{\mathrm{us}}-P L_{\mathrm{us}}
$$

where $P L_{u s}$ is propagation path loss for the channel between the UAV and the sensor node, $G_{u s}$ is for the total antenna gain from both sensor node and the UAV, and $P t_{0}$ is the fixed transmitting power by the nodes. Then, the maximal radio range is obtained when the receiving signal power is at its minimal requirement (that is, when information is still decodable, and $\left.P r_{\text {us }}=P r_{\text {min }}\right)$. The channel propagation path loss is at its maximum, that is $P L_{\mathrm{us}}=P L_{\mathrm{max}}$, and is given by:

$$
P L_{\text {max }}=P t_{0}+G_{\mathrm{us}}-P r_{\text {min }}
$$

where $P r_{\text {min }}$ is the minimal allowed receiving signal power in $\mathrm{dBm}$. As the channel between the UAV and sensor nodes is assumed to be Line-Of-Sight (LOS), the distance in meters between the UAV and a sensor node $L_{\mathrm{us}}$ is related to the path loss $P L_{\mathrm{us}}$ by [7]

$$
P L_{\mathrm{us}}=-147.55+20 \log f+20 \log L_{\mathrm{us}}
$$

where $f$ is the radio frequency in Hertz. The maximum distance $L_{\mathrm{us}}$, which we will denote $L_{\max }$, is found using $P L_{\mathrm{us}}=P L_{\max }$ in (6) with $P L_{\max }$ from equations (5).

2) Adjustment of transmitting power for sensor nodes: To establishing a continuous communication channel between a sensor node and the UAV the transmitting power of the sensor nodes will be adjusted. When the receiving signal strength is higher than the mimimal required $P r_{\min }$, there is no need for transmission power adjustment. If it is lower the transmitting power $P t$ must be adjusted to satisfy:

$$
P t=P L_{\mathrm{us}}+P r_{\min }-G_{\mathrm{us}}
$$

where $P L_{\mathrm{us}}$ is the path loss from the equation (6) with the actual distance $L_{\mathrm{us}}$ between the sensor node and the UAV. This distance can be estimated by the nodes based on a broacasting position message from the UAV.

\section{Channel capacity and energy consumption}

Considering the scenarios in this paper, the UAV and sensor nodes are usually in LOS but a signal obstacle is often occurring during data perception period for instance caused by the airframe or other auxiliary components on the UAV. The Packet-Error-Rate (PER), is therefore instantaneously changing and dependent on actual Signal-to-Noise Ratio (SNR). To estimate the data amount perceived by the UAV from a node, the channel bandwidth and PER need to be calculated. The average PER can be estimated as follows [8]:

$$
P E R_{\bar{\gamma}}=1-e^{-\frac{a_{N}}{\bar{\gamma}}} \Gamma\left(1+\frac{b_{N}}{\bar{\gamma}}\right)
$$

with

$$
a_{N}=\frac{\log _{10}\left(L_{p} c_{m}\right)}{k_{m}}, \quad b_{N}=\frac{1}{k_{m}}
$$

where $\bar{\gamma}$ is the average $\operatorname{SNR}, \Gamma($.$) is the standard gamma$ distribution function, $L_{p}$ is the packet length used in data transmission protocol in bits, and $c_{m}$ and $k_{m}$ are constants depending on the modulation method applied for signal transmission (for instance $k_{m}=\frac{1}{2}$ and $c_{m}=\frac{1}{2}$ for Frequency Shift Keying (FSK) modulation $k_{m}=2$ and $c_{m}=1$ if the modulation is Binary Phase Shift Keying (BPSK)). If $Q$ is the number of packet retransmissions due to erroneous bits, the average PER for data received from sensor $s$ at the UAV at a time $t$ becomes

$$
P E R_{u, s, t}=1-\left(1-P E R_{\bar{\gamma}}\right)^{Q}
$$

The average BER for the channel between the sensor $s$ and the UAV $u$ at a time $t$ with erroneous bits retransmission is given by

$$
B E R_{u, s, t}=1-\left(1-P E R_{u, s, t}\right)^{L_{p} / Q}
$$

Because the channel between the UAV and sensor nodes is fast varying in the time domain, the channel capacity can be described, see [8], as

$$
C_{u, s, t}=B W \int_{0}^{\infty} \log (1+t) \frac{1}{\bar{\gamma}} e^{\frac{-t}{\bar{\gamma}}} d t
$$

where $B W$ is the data bandwith. If $T$ is the time duration where a sensor node $s$ has data communication with the UAV at a moment $t$, then the received data at the UAV can be written as

$$
D_{u, s, t, T}=\int_{0}^{T} C_{u, s, t}\left(1-B E R_{u, s, t}\right) d t
$$

As the sensors might not be visited by the UAV very often, we will assume that each sensor will use all of this period $T$ for transmitting sensing data during each visit. The total data received by the UAV from all sensors in the network is

$$
D_{\text {total }}=\sum_{i=1}^{n} \sum_{j=1}^{N_{i}} D_{u, i, j, T}
$$

where $N_{i}$ is the number of sensors in the network and the number of times that the UAV has data communications with a sensor node $i$.

\section{Particle SWARm Optimization Algorithm}

Particle Swarm Optimization (PSO) is a stochastic population-based optimization method proposed in [9]. Communications and networking is an area where PSO has been applied widely to solve network performance optimization problems [10] [11] [12]. PSO is also used for wireless sensor networks to effectively create clusters of nodes where data collection, energy consumptions and network lifetime is optimized, see [12] and [13]. It can also be used to provide a path planning solution for one UAV in data collection from a wide-area sensor network [4].

In this paper, PSO is used to find the best waypoints for a flyable UAV path when the sensor nodes are drifting on the ocean surface. In the PSO algorithm, the following objectives are taken into account: a) Total energy consumption by sensor network; b) total data received by the UAV from the sensor nodes during its flight; c) total flight time for the UAV and d) 
the freshness of sensing data from sensor nodes. The waypoints are chosen based on optimizing the objective function

$$
f=\alpha_{1} f_{E}+\alpha_{2} f_{D}+\alpha_{3} f_{T}+\alpha_{4} f_{F}
$$

where $\alpha_{1}, \alpha_{2}, \alpha_{3}$ and $\alpha_{4}$ are positive scalar weighting constants, and $f_{E}, f_{D}, f_{T}$ and $f_{F}$ are functions described in detail in the following.

\section{A. Total energy consumption on sensors, $f_{E}$}

This value for different scenarios are explained in the following.

1) Scenarios 2 \& 3: Each node will transmit its data at constant transmitting power $P t_{0}$. Assuming that once a node transmits data, it will occur in an entire period of $T$, hence, the total energy consumption for sensor network is

$$
E_{\text {total }}^{S}=\sum_{i=1}^{N_{S}} N_{i} P t_{0}
$$

2) Scenario 1: Each node will transmit its data at a transmitting power $P t_{u, s, t}$ which depends on the distance to the UAV (defined in (7)).

$$
E_{\text {total }}^{S}=\sum_{i=1}^{N_{S}} \sum_{j=1}^{N_{i}} T P t_{u, j, t}
$$

\section{B. Total data received, $f_{D}$}

Equation (14) represents the total amount of data received by the UAV from the sensor nodes during its flight.

\section{Flight time of the UAV, $f_{T}$}

This is the time required for the UAV to fly along its trajectory with the calculated waypoints. The trajectory is generated using a Dubins path through these waypoints, [14]. A Dubins path is composed of a sequence of three motions: Straight, and Left or Right at the maximum steering angle of the vehicle. The total flight time $f_{T}$ (15) is assumed proportional to the length of the Dubins path and the UAV has a constant ground speed in the entire simulation period.

\section{The freshness of collected data, $f_{F}$}

In general, a sensor node will try to transmit sensing data periodically and if the UAV is within its communication range the UAV may receive some data from this node. Due to the UAV's mobility and other obstacles, the data can not always be received correctly because of the fast time-varying channel between the UAV and the node. In such cases, the node needs to wait for the next visit by the UAV; and this node will have a higher priority when a data communication possibility with the UAV opens again. In the simulations, this priority is equal to the waiting time of the node since from the last visited by the UAV.
TABLE I. PARAMETERS FOR PSO, UAV AND DATA TRANSMISSION

\begin{tabular}{l|c|l|c}
\hline Parameter & Value & Parameter & Value \\
\hline$n$ & 4 & $N^{\mathrm{WP}}$ & 10 \\
\hline$h_{u}$ & $120 \mathrm{~m}$ & $v_{u}$ & $10 \mathrm{~m} / \mathrm{s}$ \\
\hline$G_{\mathrm{i}}$ & $10 \mathrm{dBi}$ & $G_{\mathrm{u}}$ & $10 \mathrm{dBi}$ \\
\hline$G_{\mathrm{j}}$ & $10 \mathrm{dBi}$ & $l_{\text {ctr }}$ & $20 \mathrm{bits}$ \\
\hline$P t_{0}$ & $5 \mathrm{~mW}$ & $f$ & $5.8 \mathrm{GHz}$ \\
\hline$B$ & $5 \mathrm{MHz}$ & $P r_{\min }$ & $-90 \mathrm{dBm}$ \\
\hline$x_{0}$ & $\pm 150 \mathrm{~m}$ & $y_{0}$ & $\pm 150 \mathrm{~m}$ \\
\hline$I_{0}$ & $-95 \mathrm{dBm}$ & $Q$ & 5 \\
\hline$c_{m}$ & 1 & $k_{m}$ & 2 \\
\hline$L_{p}$ & $20 \mathrm{bytes}$ & $L_{\max }$ & $355 \mathrm{~m}$ \\
\hline$T$ & $10 \mathrm{~s}$ & $N^{\text {Iterations }}$ & 5000 times \\
\hline$E_{0}$ & $1000 \mathrm{Joule}$ & Simulations & $10000 \mathrm{~s}$ \\
\hline$\alpha_{1}$ & 5 & $\alpha_{2}$ & $10^{7}$ \\
\hline$\alpha_{3}$ & $10^{-2}$ & $\alpha_{4}$ & 1 \\
\hline \multicolumn{4}{|l}{}
\end{tabular}

\section{Simulation}

Table I shows the list of parameters that are used in simulations for performance comparison between the scenarios. $L_{\text {ctr }}$ is the number of control bits in data protocol; $x_{0}$ and $y_{0}$ are the coordinate ranges where the sensor nodes' initial positions have a uniform distribution; $h_{u}, v_{u}$ are the altitude and ground-speed of the UAV. In the case of Scenario 3 where the velocity vector is measured, the update period is assumed to be the same as the position update, $T$. The actual SNR will be calculated based on the difference between the actual receiving signal strength and its minimal, allowed value $\left(P r_{\min }\right)$. For the values of weighting constants $\left(\alpha_{1}\right.$ to $\left.\alpha_{4}\right)$ in the fitness function (15), these were selected such that the influence of each cost is approximately normalized. The values given in Table I where used in the simulations.

There are two main evaluations for each scenario. The first one shows the trajectory of the UAV and the nodes' movements during the simulation period. For a sensor node, there are two points that represent its actual position and estimated position. In such plots, there are also lines connecting the UAV and a sensor node when they are within communication range. The other plot shows the measurement error for nodes positions in Scenario 1, 2 and nodes position estimation error in Scenario 3 .

\section{A. Scenario 1: Full sensor node position information}

In this case, the UAV can communicate and has the node's positions in every period $T$. As it may use a high energy consumption on the sensor nodes, a plot for total energy consumption per node will be shown and used for a comparison with that in other cases that the nodes only transmit at fixed transmitting power. Figure 1 shows that there is still a small difference between actual node positions and their measured values due to the period position transmission by the sensor nodes in this case is $T=10 \mathrm{~s}$. This is visualized in Figure 2 where the maximal difference is approximated at 50 meters. This variation will be reduced when reducing the transmission period but it will consequently increase energy consumption for the nodes. Regarding energy consumption, with the transmission period of $10 \mathrm{~s}$ as in Table I, Figure 3 shows that after nearly 3 hours flight simulation time, a node needs to use an energy varies between 70 and 100 Joule, which has an average value of 90 Joule per sensor. If reducing this period for instance to $1 \mathrm{~s}$, then the energy amount consumed per sensor node becomes approximated at 900 Joule in average. 


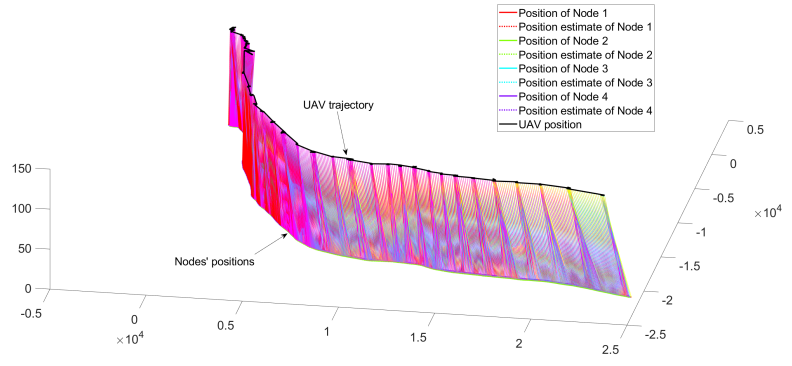

Fig. 1. Scenario 1: Position of sensor nodes and optimized UAV trajectory

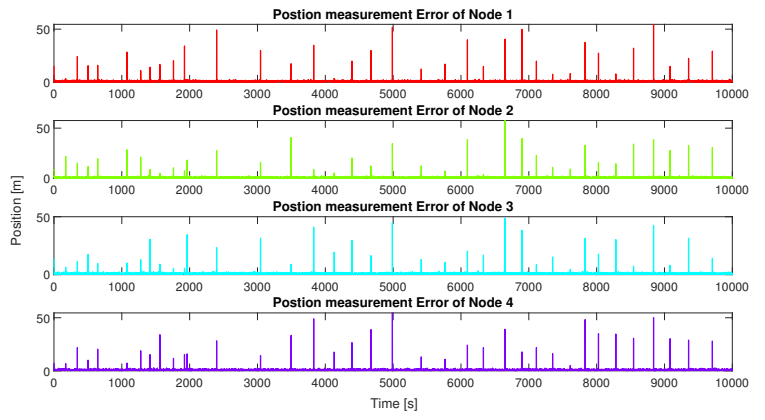

Fig. 2. Scenario 1: Difference between actual position of the sensor nodes, and the last information about the postion which is used in the trajectory planning for the UAV.

In that case, the node position error will be much minized and it can say that the UAV has full information of the nodes. The sensor nodes will however run out of battery just after a short time of operation. This is a major constraint of implementing this scenario for the network.

\section{B. Scenario 2: Range-limited position information}

Here, the nodes' positions and sensor data will be transmitted to the UAV only if a communication link is established at intervals with period $T$. The trajectory of the UAV will therefore depend on the last update on positions of the nodes. Due to the nodes mobility, there might be a large deviation between the actual nodes' positions and the information used

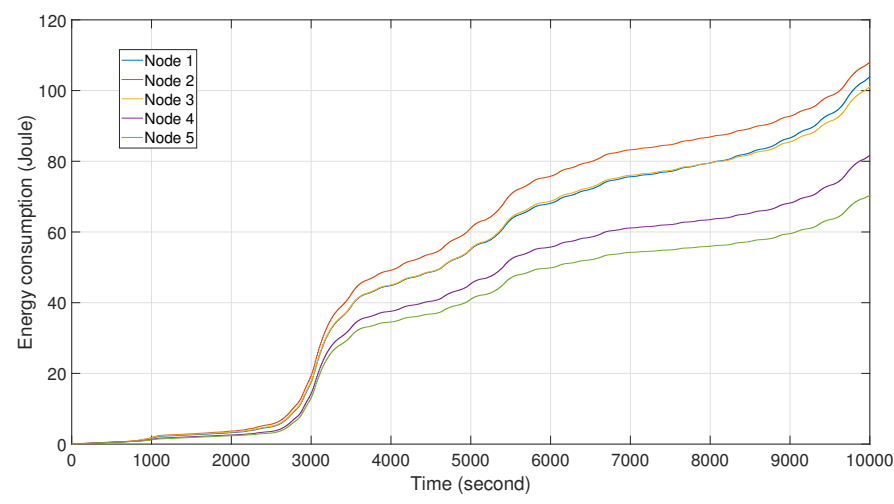

Fig. 3. Scenario 1: Energy consumption per sensor node when adapting transmission power.

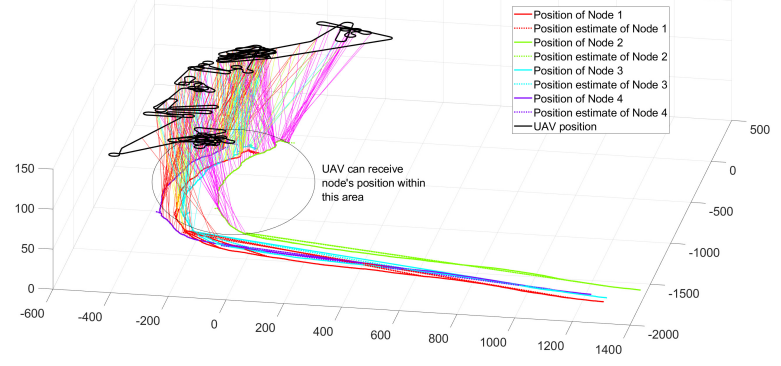

Fig. 4. Scenario 2: Position of sensor nodes and optimized UAV trajectory.

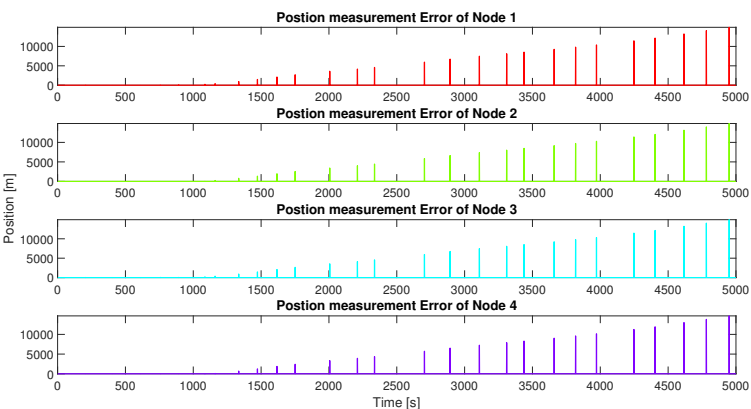

Fig. 5. Scenario 2: Difference between actual position of the sensor nodes, and the last information about the postion which is used in the trajectory planning for the UAV.

for planning. This will affect the quality of data collected, both in terms of the total data received and the freshness of the data received from the network. Figure 4 shows the nodes' motion and the UAV trajectory for this scenario. Only for the first 15 minutes the UAV is able to receive position information from all sensor nodes regularly. After this period, the error between the actual nodes' positions and the last updates starts to increase sharply and the UAV starts to lose track of some nodes. As the UAV only continues to fly over the last known position errors continue to increase as shown in Figure 5. As an example, after a simulation time of 1.5 hours, the nodes' position errors can increase up to $10 \mathrm{~km}$. The average energy consumption for one node is approximately 0.9 (Joule), which is around 100 times less than that for the first case.

\section{Scenario 3: Estimation-based position information}

To improve knowledge about the nodes' position, while still maintaining a low energy consumption, a KF is applied in this scenario. Notice is that the UAV will still need to be within communication range of a node for a measurement update of the KF. This scenario will consider two measurement models, that is, with and without the velocity vector measurements as mentioned in Section III-B.

1) Without velocity vector measurement: The application of KF has doubled the time period for the UAV to have good information on the nodes movements compared to that in the secenario 2 (See Figure $6 \& 4$ ). The errorness of node position measurements is ten times smaller (See Figure $7 \& 5$ ) than that in the scenario 2 but its unacurracy still gradually causes the 


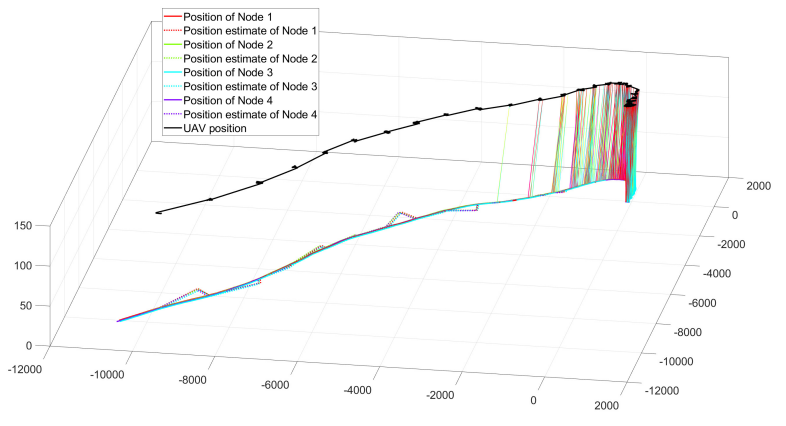

Fig. 6. Scenario 3 - Case 1: Position of sensor nodes and optimized UAV trajectory.

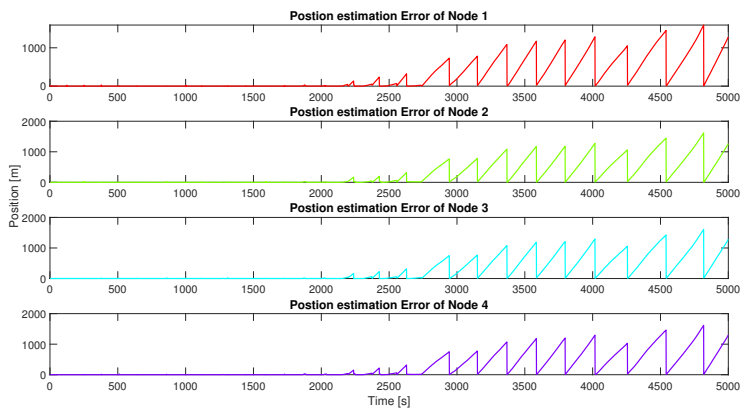

Fig. 7. Scenario 3 - Case 1: Position estimation error using a KF without velocity vector measurements.

UAV's trajectory far from actual locations of the nodes (See Figure 6). The UAV still loses the sensor nodes even though this happens later than when it occurred in Scenario 2.

2) With velocity vector measurement: If there is a possibility to update the velocity vector onboard the UAV, the KF significantly improves the position estimation errors. Figure 9 shows that the maximal position error is quite similar to what was achieved in Scenario 1 (compare with Figure 2). The accurate estimation allows the UAV to continuously follow the movements of the sensor nodes, see Figure 8.

\section{CONCLUSION}

The application of a Kalman filter with velocity vector measurements outperforms the other two scenarios both with

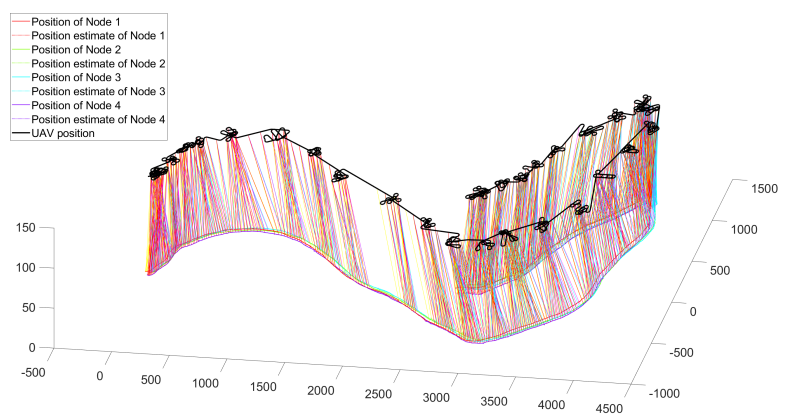

Fig. 8. Scenario 3 - Case 2: Position of sensor nodes and optimized UAV trajectory.

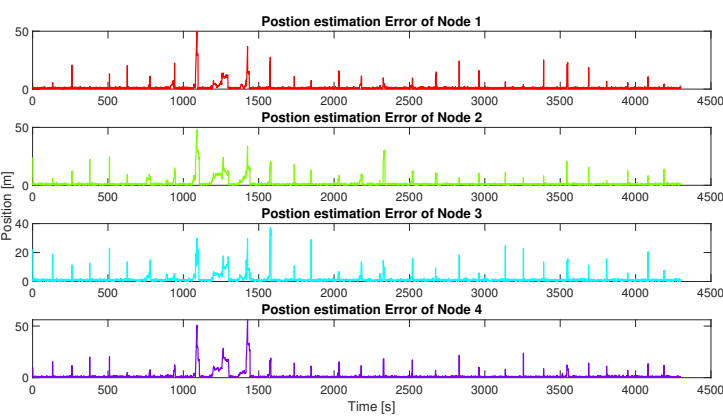

Fig. 9. Scenario 3 - Case 2: Position estimation error using a KF with velocity vector measurements.

respect to energy consumption on the sensor nodes and to the UAVs ability to follow the nodes' movements. Specifically, this scenario gives the same prediction for the nodes' movement as achieved with adaptive transmission power, but it leads to significantly lower energy consumption on the nodes. One of the main challenges of this approach might be to realize velocity vector measurements. It is also unclear if a linear model used in the Kalman filter can sufficiently well approximate the nodes motion in a real world scenario. These are aspects that will be investigated in future work. We will also consider the use of multiple UAVs for data collection from a sensor network with increased spatial distribution.

\section{ACKNOWLEDGMENT}

This work is sponsored by the Research Council of Norway through the Centres of Excellence funding scheme, project number 223254 - Centre for Autonomous Marine Operations and Systems (NTNU-AMOS).

\section{REFERENCES}

[1] H. C. Bittig, T. Steinhoff, H. Claustre, B. Fiedler, N. L. Williams, R. Sauzède, A. Körtzinger, and J.-P. Gattuso, "An alternative to static climatologies: Robust estimation of open ocean $\mathrm{CO} 2$ variables and nutrient concentrations from $\mathrm{T}, \mathrm{S}$, and $\mathrm{O} 2$ data using bayesian neural networks," Frontiers in Marine Science, vol. 5, p. 328, 2018. [Online]. Available: https://www.frontiersin.org/article/10.3389/fmars.2018.00328

[2] P. Bresnahan, T. Martz, Y. Takeshita, K. Johnson, and M. Lashomb, "Best practices for autonomous measurement of seawater $\mathrm{pH}$ with the honeywell durafet," Methods in Oceanography, vol. 9, pp. 44-60, 08 2014.

[3] B. Li, Z. Fei, and Y. Zhang, "Uav communications for $5 \mathrm{~g}$ and beyond: Recent advances and future trends," IEEE Internet of Things Journal, vol. 6, no. 2, pp. 2241-2263, 2019.

[4] T. D. Ho, E. I. Grøtil, P. B. Sujit, T. A. Johansen, and J. B. Sousa, "Optimization of wireless sensor network and uav data acquisition," Journal of Intelligent and Robotic Systems, vol. 78, p. 159-179, Mar. 2015.

[5] P. G. Radoglou, P. Sarigiannidisa, T. Lagkasbc, and M. Ioannis, "A compilation of UAV applications for precision agriculture," Computer Networks, vol. 172, no. 107148, May 2020.

[6] D. T. Ho, E. I. Grøtli, and T. A. Johansen, "Heuristic algorithm and cooperative relay for efficient energy data collection with a uav," in Proc. of the IEEE International Conference on Computing, Management \& Telecommunications, Ho Chi Minh, Vietnam, 2013.

[7] A. Goldsmith, Path Loss and Shadowing. Cambridge University Press, 2005, p. 27-63.

[8] - Capacity of Wireless Channels. Cambridge University Press, 2005 , p. $99-125$ 
[9] J. Kennedy and R. Eberhart, "Particle swarm optimization," in Proceedings of ICNN'95 - International Conference on Neural Networks, vol. 4, 1995, pp. 1942-1948 vol.4.

[10] W. Li, "PSO based wireless sensor networks coverage optimization on dems," in Advanced Intelligent Computing Theories and Applications. With Aspects of Artificial Intelligence. Springer, 2012, pp. 371-378.

[11] Y. Yang, B. Li, and B. Ye, "Wireless sensor network localization based on pso algorithm in nlos environment," in 2016 8th International Conference on Intelligent Human-Machine Systems and Cybernetics (IHMSC), vol. 01, 2016, pp. 292-295.

[12] S. Jiang, "Leach protocol analysis and optimization of wireless sensor networks based on pso and ac," in 2018 10th International Conference on Intelligent Human-Machine Systems and Cybernetics (IHMSC), vol. 02, 2018, pp. 246-250.

[13] C. Huang and J. Fei, "Uav path planning based on particle swarm optimization with global best path competition," International Journal of Pattern Recognition and Artificial Intelligence, vol. 32, 102017.

[14] A. M. Shkel and V. Lumelsky, "Classification of the dubins set," Robotics and Autonomous Systems, vol. 34, no. 4, pp. 179 - 202, 2001. [Online]. Available: http://www.sciencedirect.com/science/article/pii/S0921889000001275 